\title{
A Generalized Inverse Binomial Summation Theorem and Some Hypergeometric Transformation Formulas
}

\author{
S. M. Ripon \\ Department of Mathematics, University of Central Florida, Orlando, FL, USA \\ Correspondence should be addressed to S. M. Ripon; smripon@knights.ucf.edu
}

Received 17 July 2016; Accepted 24 August 2016

Academic Editor: Toufik Mansour

Copyright (C) 2016 S. M. Ripon. This is an open access article distributed under the Creative Commons Attribution License, which permits unrestricted use, distribution, and reproduction in any medium, provided the original work is properly cited.

A generalized binomial theorem is developed in terms of Bell polynomials and by applying this identity some sums involving inverse binomial coefficient are calculated. A technique is derived for calculating a class of hypergeometric transformation formulas and also some curious $q$ series identities.

\section{Introduction}

Some of the most recent developments are on the use of different techniques for obtaining sums of hypergeometric series. In this paper, we present a new method for calculating the following summations and also a generalized theorem related to these series is investigated. We investigated the following summations formula with some restrictions for the functions $f(k, y)$ :

$$
\begin{gathered}
\sum_{k=1}^{\infty} \frac{(-1)^{m k} x^{k+n}}{\left[(k)_{n+1}\right]^{m}} f(k, y), \\
\sum_{k=1}^{\infty} \frac{(-1)^{m k} x^{k+n}}{\left[(k)_{n+1}\right]^{m}} .
\end{gathered}
$$

We assume that the function $f(k-n, y)$ has no poles at $(n-k+l)$, where $l$ is an integer ranged from $1 \leq l \leq$ $k$.
As it turns out, the above summation formula for the constant function $f(k, y)=1$ gives us a new generalized hypergeometric transformation formula of the type

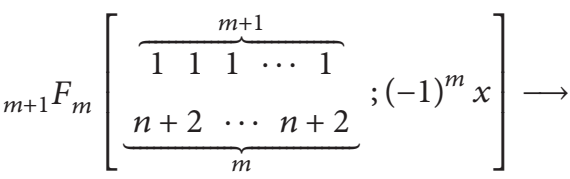

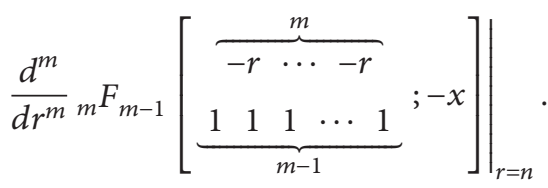

We further investigated some $q$ series closely related to the famous Roger-Ramanujan identities.

$$
\sum_{v=1}^{\infty} \frac{(q)_{v-1}}{(q)_{r+v}}\left(a q^{r}\right)^{v} .
$$


In our present investigation dealing with the series identity, we shall also make use of other such higher transcendental functions, the Riemann Zeta function and Hurwitz Zeta function, which are defined by

$$
\begin{gathered}
\zeta(s)=\sum_{k=1}^{\infty} \frac{1}{k^{s}}, \\
\zeta(s, a+1)=\sum_{k=1}^{\infty} \frac{1}{(k+a)^{s}}=\zeta(s)-H_{a}^{(s)},
\end{gathered}
$$

where $H_{a}^{(s)}$ is the generalized harmonic numbers defined by

$$
H_{a}^{(s)}=\sum_{k=1}^{a} \frac{1}{k^{s}}=\frac{(-1)^{s}}{\Gamma s}\left[\psi^{(s-1)}(1)-\psi^{(s-1)}(a+1)\right]
$$

$$
\text { for } s \geq 1, a \geq 0 \text {. }
$$

In the above identity we used the $\psi$ notation for denoting the generalized polygamma function of order $m$ which is given by the $m+1$ th times logarithmic derivative of Gamma function

$$
\begin{aligned}
& \psi^{(m)}(z)=\frac{d^{m}}{d z^{m}} \psi^{(1)}(z)=\frac{d^{m+1}}{d z^{m+1}} \ln \Gamma(z) ; \\
& \psi(1)=-\gamma .
\end{aligned}
$$

$\gamma$ denotes the famous Euler-Mascheroni constant. The derivatives of generalized harmonic number are given:

$$
\frac{d^{m}}{d n^{m}} H_{n}^{(p)}=(-1)^{m+1} \frac{\Gamma(m+p)}{\Gamma(p)} \zeta(m+p, n+1) .
$$

And we used $(\lambda)_{v}$ for the Pochhammer symbol defined (for $\lambda, v \in C$ and in terms of the Gamma function) by

$$
(\lambda)_{v}=\frac{\Gamma(\lambda+v)}{\Gamma(\lambda)}=\lambda(\lambda+1) \cdots(\lambda+v-1) ;
$$

$$
(\lambda)_{0}=1
$$

The generalized hypergeometric function is defined by

$$
\begin{aligned}
{ }_{p} F_{q} & {\left[\begin{array}{llll}
a_{1} & a_{2} & \cdots & a_{p} \\
b_{1} & b_{2} & \cdots & b_{q}
\end{array}\right] z } \\
= & \sum_{k=0}^{\infty} \frac{\left(a_{1}\right)_{k}\left(a_{2}\right)_{k} \cdots\left(a_{p}\right)_{k}}{\left(b_{1}\right)_{k}\left(b_{2}\right)_{k} \cdots\left(b_{q}\right)_{k}} \frac{z^{k}}{k !} .
\end{aligned}
$$

The notation for generalized hypergeometric functions was introduced by Pochhammer in 1870 and modified by Barnes [1] and later by Maier and Slater [2]. A number of notational variations are commonly used. Most common notation is introduced by Graham et al. [3] using square brackets and a semicolon.

The complete Bell polynomials of order $n$ are defined as

$$
\begin{aligned}
Y_{n}\left[x_{1}, x_{2}, \ldots, x_{n}\right]= & \sum_{k_{1}+2 k_{2}+3 k_{3}+\cdots+n k_{n}=n} \frac{n !}{k_{1} ! k_{2} ! \cdots k_{n} !} \\
& \cdot\left(\frac{x_{1}}{1 !}\right)^{k_{1}}\left(\frac{x_{2}}{2 !}\right)^{k_{2}} \cdots\left(\frac{x_{n}}{n !}\right)^{k_{n}} .
\end{aligned}
$$

First few terms of these polynomials can be derived by

$$
\begin{aligned}
Y_{1}\left[x_{1}\right] & =x_{1}, \\
Y_{2}\left[x_{1}, x_{2}\right] & =x_{1}^{2}+x_{2}, \\
Y_{3}\left[x_{1}, x_{2}, x_{3}\right] & =x_{1}^{3}+3 x_{1} x_{2}+x_{3} .
\end{aligned}
$$

\section{Main Results}

Theorem 1. For integers $m>0, n \geqslant 0$,

$$
\begin{aligned}
& \sum_{k=1}^{\infty} \frac{(-1)^{m k} x^{k+n}}{\left[(k)_{n+1}\right]^{m}} f(k, y)=\frac{(-1)^{m}}{m !(n !)^{m}} F_{m}^{(m)}(n, x, y) \\
& \quad-\sum_{k=0}^{n} \sum_{j=0}^{m} \frac{(-1)^{j} x^{k}}{m !(n !)^{m}}\left(\begin{array}{l}
n \\
k
\end{array}\right)^{m}\left(\begin{array}{c}
m \\
j
\end{array}\right) f^{(m-j)}(k-n, y) \\
& \quad \cdot Y_{m}\left[m B_{1}, \ldots, m B_{m}\right],
\end{aligned}
$$

where $\left[F_{m}(r, x, y)=\sum_{k=0}^{\infty} x^{k}\left(\begin{array}{l}r \\ k\end{array}\right)^{m} f(k-r, y)\right.$ and $F_{m}^{(m)}(n, x, y)=\left.\left(d^{m} / d r^{m}\right) F_{m}(r, x, y)\right|_{r=n}$.

Proof. Let us define a function $F_{m}(r, x, y)$ where $r, x, y \in \mathbb{R}$ such that

$$
F_{m}(r, x, y)=\sum_{k=0}^{\infty} x^{k}\left(\begin{array}{l}
r \\
k
\end{array}\right)^{m} f(k-r, y) .
$$

We also assume the function $f(k-r, y)$ has no poles at $(n-$ $k+l)$ for each $l$ and $m$ such that

$$
1 \leqslant l \leqslant k, \quad m \in \mathbb{Z}^{+} .
$$

We introduce another notation for the $m$ th derivative with respect to $r$ of the defined function $F_{m}(r, x, y)$ :

$$
F_{m}^{(m)}(n, x, y)=\left.\frac{d^{m}}{d r^{m}} F_{m}(r, x, y)\right|_{r=n} .
$$

Furthermore, we see the series also defined for $r$. It can be extended to the interval $[0, \infty]$, because when $k \rightarrow(r+l)$, where $r$ is a positive integer, the further terms of the series just vanish.

$$
\lim _{k \rightarrow(r+l)}\left[\left(\begin{array}{l}
r \\
k
\end{array}\right)^{m} f(k-r, y)\right]=0 .
$$

Making use of certain special properties of Bell polynomials we can evaluate successive derivative of a given function. Let us consider a function $f(x, y, z)$ which has a Taylor series expansion around $x$; the detailed procedure of these kinds is extensively discussed in the paper [4]. Following the same process discussed in $[5,6]$ we can write the following elegant identity by virtue of Bell polynomials:

$$
\begin{aligned}
& \frac{d^{n}}{d r^{n}} e^{f(r, x, y)}=e^{f(r, x, y)} Y_{n}\left[f^{1}(r, x, y), f^{2}(r, x, y), \ldots,\right. \\
& \left.f^{n}(r, x, y)\right] .
\end{aligned}
$$


The following identity can be recovered from the same method used in [5, page 8$]$ :

$$
\begin{aligned}
& \frac{d^{j}}{d r^{j}}\left(\begin{array}{l}
r \\
k
\end{array}\right)^{m}=\left(\begin{array}{l}
r \\
k
\end{array}\right)^{m} Y_{j}\left[m\left(H_{r}-H_{r-k}\right),\right. \\
& \left.\quad m\left(H_{r}^{1}-H_{r-k}^{1}\right), \ldots, m\left(H_{r}^{j-1}-H_{r-k}^{j-1}\right)\right] .
\end{aligned}
$$

$H_{r}^{j}$ represents the $j$ th order derivative harmonic number $H_{r}^{(1)}$ with respect to $r$. The derivative of harmonic numbers can be evaluated by using the formula given in

$$
\begin{aligned}
H_{r}^{j-1}-H_{r-k}^{j-1} & =\left.\frac{d^{j-1}}{d r^{j-1}}\left(H_{r}^{(1)}-H_{r-k}^{(1)}\right)\right|_{r=n} \\
& =(-1)^{j-1} \Gamma(j)\left(H_{n}^{(j)}-H_{n-k}^{(j)}\right)=B_{j} .
\end{aligned}
$$

Hence, upon considering (18) and (19), we find

$$
\left.\frac{d^{j}}{d r^{j}}\left(\begin{array}{l}
r \\
k
\end{array}\right)^{m}\right|_{r=n}=\left(\begin{array}{l}
n \\
k
\end{array}\right)^{m} Y_{j}\left[m B_{1}, m B_{2}, \ldots, m B_{j}\right] .
$$

The above identity was derived extensively in [5] and a modified version was calculated in [7]. Now differentiating (13) $m$ times with respect to $r$ and considering the case $r=n$, where $r$ is a positive integer as well as using the definition from (15), we can derive

$$
\begin{gathered}
F_{m}^{(m)}(n, x, y)=\left.\frac{d^{m}}{d r^{m}} F_{m}(r, x, y)\right|_{r=n} \\
=\left.\sum_{k=0}^{\infty} \sum_{j=0}^{m} x^{k}\left(\begin{array}{c}
m \\
j
\end{array}\right) \frac{d^{j}}{d r^{j}}\left(\begin{array}{l}
r \\
k
\end{array}\right)^{m}\right|_{r=n} \\
\left.\cdot \frac{d^{m-j}}{d r^{m-j}} f(k-r, y)\right|_{r=n} .
\end{gathered}
$$

Making use of the identity derived in (20) we find

$$
\begin{aligned}
& F_{m}^{(m)}(n, x, y)=\sum_{k=0}^{n} \sum_{j=0}^{m} x^{k}\left(\begin{array}{l}
m \\
j
\end{array}\right) f^{m-j}(k-r, y)\left(\begin{array}{l}
n \\
k
\end{array}\right)^{m} \\
& \cdot Y_{j}\left[m B_{1}, m B_{2}, \ldots, m B_{j}\right] \\
& +\sum_{k=n+1}^{\infty} \sum_{j=0}^{m} x^{k}\left(\begin{array}{c}
m \\
j
\end{array}\right) f^{m-j}(k-r, y)\left(\begin{array}{l}
n \\
k
\end{array}\right)^{m} \\
& \cdot Y_{j}\left[m B_{1}, m B_{2}, \ldots, m B_{j}\right] .
\end{aligned}
$$

From the asymptotic properties of Bell polynomials we have

$$
\begin{aligned}
Y_{j} & {\left[f\left(\frac{1}{x}\right), f^{2}\left(\frac{1}{x}\right), \ldots, f^{j}\left(\frac{1}{x}\right)\right] } \\
& =f^{j}\left(\frac{1}{x}\right) \sum_{k_{1}+2 k_{2}+\cdots+j k_{j}=j} G\left(k_{1}, k_{2}, \ldots, k_{j}\right) .
\end{aligned}
$$

Considering the above property in (23), for $m \geq 1$ and $n, l \in Z^{+}$are both positive integers, we can readily evaluate the following limits:

$$
\begin{aligned}
& \lim _{k \rightarrow(n+l)}\left(\begin{array}{l}
n \\
k
\end{array}\right)^{m} Y_{1}\left[m \Gamma(1)\left(H_{n}^{(1)}-H_{n-k}^{(1)}\right)\right]=0, \\
& \lim _{k \rightarrow(n+1)}\left(\begin{array}{l}
n \\
k
\end{array}\right)^{m} Y_{2}\left[m \Gamma(1)\left(H_{n}^{(1)}-H_{n-k}^{(1)}\right),\right. \\
& \left.-m \Gamma(2)\left(H_{n}^{(2)}-H_{n-k}^{(2)}\right)\right]=0 .
\end{aligned}
$$

Considering next few cases, we can move to final relation

$$
\lim _{k \rightarrow(n+1)}\left(\begin{array}{l}
n \\
k
\end{array}\right)^{m} Y_{m-1}\left[m B_{1}, m B_{2}, \ldots, m B_{m-1}\right]=0
$$

where $B_{m}$ is defined as in (19). Recalling the properties of Bell polynomials mentioned in (25) if we consider the limit $k \rightarrow$ $(n+l)$ for $l=1,2, \ldots, \infty$ we observe that for every $0 \leq j \leq m$ the limiting value vanishes but it surprisingly gives us limiting value for $j=m$.

$$
\begin{aligned}
& \lim _{k \rightarrow(n+l)} \sum_{j=0}^{m} x^{k}\left(\begin{array}{l}
m \\
j
\end{array}\right) f^{m-j}(k-r, y)\left(\begin{array}{l}
n \\
k
\end{array}\right)^{m} \\
& \cdot Y_{j}\left[m B_{1}, \ldots, m B_{j}\right]=0 \\
& +\lim _{k \rightarrow(n+l)}\left[x^{k}\left(\begin{array}{l}
m \\
m
\end{array}\right) f^{m-m}(k-r, y)\left(\begin{array}{l}
n \\
k
\end{array}\right)^{m}\right. \\
& \left.\cdot Y_{m}\left[m B_{1}, m B_{2}, \ldots, m B_{m}\right]\right]=x^{n+l} f(l, y) \\
& \cdot \lim _{k \rightarrow(n+l)}\left[\left(\begin{array}{l}
n \\
k
\end{array}\right)^{m} Y_{m}\left[m B_{1}, m B_{2}, \ldots, m B_{m}\right]\right] .
\end{aligned}
$$

Furthermore, expanding the product of generalized harmonic numbers in asymptotic form with Big $O$ notation, we can further deduce

$$
\begin{gathered}
\left(H_{n}^{(1)}-H_{n-k}^{(1)}\right)^{m_{1}}\left(H_{n}^{(2)}-H_{n-k}^{(2)}\right)^{m_{2}} \cdots\left(H_{n}^{(i)}-H_{n-k}^{(i)}\right)^{m_{i}} \\
=\frac{1}{(n-k+1)^{u}}+O\left(\frac{1}{(n-k+1)^{v}}\right)+\cdots
\end{gathered}
$$

with $u=m_{1}+2 m_{2}+\cdots+i m_{i}$ and $m_{1}+2 m_{2}+\cdots+i m_{i}>v$. Substituting the above asymptotic identity in (26) we find

$$
\begin{aligned}
& \lim _{k \rightarrow(n+l)}\left[\left(\begin{array}{l}
n \\
k
\end{array}\right)^{m} Y_{m}\left[m B_{1}, \ldots, m B_{m}\right]\right]=\lim _{k \rightarrow(n+l)}\left(\begin{array}{l}
n \\
k
\end{array}\right)^{m} \\
& \cdot\left\{\frac{1}{(n-k+1)^{m}}+O\left(\frac{1}{(n-k+1)^{m-1}}\right)+\cdots\right\} \\
& \cdot Y_{m}\left[m \Gamma(1), \ldots,(-1)^{m-1} m \Gamma(m)\right] .
\end{aligned}
$$


After some tedious calculations we find

$$
\begin{aligned}
& \lim _{k \rightarrow(n+l)}\left[\left(\begin{array}{l}
n \\
k
\end{array}\right)^{m} Y_{m}\left[m B_{1}, \ldots, m B_{m}\right]\right] \\
& =\left[\frac{(-1)^{l-1} \Gamma(l) \Gamma(n+1)}{\Gamma(n+l+1)}\right]^{m} \\
& \cdot Y_{m}\left[m \Gamma(1), \ldots,(-1)^{m-1} m \Gamma(m)\right] .
\end{aligned}
$$

In view of the known identity for Bell polynomials,

$$
Y_{m}\left[m \Gamma(1), \ldots,(-1)^{m-1} m \Gamma(m)\right]=m ! .
$$

We can finally obtain a closed form for the limit

$$
\begin{aligned}
\lim _{k \rightarrow(n+l)}\left[\left(\begin{array}{l}
n \\
k
\end{array}\right)^{m} Y_{m}\left[m B_{1}, m B_{2}, \ldots, m B_{m}\right]\right] \\
=\left[\frac{(-1)^{l-1} n !}{l_{n+1}}\right]^{m} m ! .
\end{aligned}
$$

Substituting the limiting value of (26) in (31), we can readily obtain a closed form for the required limit

$$
\begin{aligned}
& \lim _{k \rightarrow(n+l)} \sum_{j=0}^{m} x^{k}\left(\begin{array}{c}
m \\
j
\end{array}\right) f^{m-j}(k-r, y)\left(\begin{array}{l}
n \\
k
\end{array}\right)^{m} \\
& \cdot Y_{j}\left[m B_{1}, m B_{2}, \ldots, m B_{j}\right]=x^{n+l} f(l, y) \\
& \cdot\left[\frac{(-1)^{l-1} n !}{l_{n+1}}\right]^{m} m ! .
\end{aligned}
$$

Applying the above limiting value, we have

$$
\begin{aligned}
& \sum_{k=n+1}^{\infty} \sum_{j=0}^{m} x^{k}\left(\begin{array}{c}
m \\
j
\end{array}\right) f^{m-j}(k-r, y)\left(\begin{array}{l}
n \\
k
\end{array}\right)^{m} \\
& \cdot Y_{j}\left[m B_{1}, m B_{2}, \ldots, m B_{j}\right]=m ! \sum_{k=1}^{\infty} x^{n+k} f(k, y) \\
& \cdot\left[\frac{(-1)^{k-1} n !}{k_{n+1}}\right]^{m}=(-1)^{m} m !(n !)^{m} \\
& \cdot \sum_{k=1}^{\infty} \frac{(-1)^{m k} x^{k+n}}{\left[k_{n+1}\right]^{m}} f(k, y) .
\end{aligned}
$$

Finally by combining (22) and (33), we finally obtain Theorem 1 .

Theorem 2. For integers $m>0, n \geqslant 0$,

$$
\begin{aligned}
\sum_{k=1}^{\infty} & \frac{(-1)^{m k} x^{k+n}}{\left[(k)_{n+1}\right]^{m}} f(k+n, y) \\
& =\frac{(-1)^{m}}{m !(n !)^{m}}\left[Q_{m}^{(m)}(n, x, y)\right. \\
& \left.-\sum_{k=0}^{n} x^{k} f(k, y)\left(\begin{array}{l}
n \\
k
\end{array}\right)^{m} Y_{m}\left[m B_{1}, m B_{2}, \ldots, m B_{m}\right]\right]
\end{aligned}
$$

where $Q_{m}(r, x, y)=\sum_{k=0}^{\infty} x^{k}\left(\begin{array}{l}r \\ k\end{array}\right)^{m} f(k, y)$ and $Q_{m}^{(m)}(n, x, y)=$ $\left.\left(d^{m} / d r^{m}\right) Q_{m}(r, x, y)\right|_{r=n}$.

Proof. The proof is similar to the previous one. Similarly as before we consider the function

$$
Q_{m}(r, x, y)=\sum_{k=0}^{\infty} x^{k}\left(\begin{array}{l}
r \\
k
\end{array}\right)^{m} f(k, y)
$$

Then by the same process and with same restrictions we can easily obtain Theorem 2 .

Theorem 3. For integers $m>0, n \geqslant 0$, and $|x|<1$,

$$
\begin{aligned}
& F_{m}^{(m)}(n, x)
\end{aligned}
$$

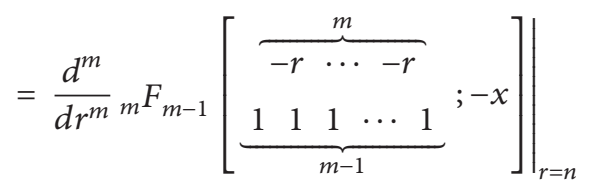

$$
\begin{aligned}
& =\frac{m !(n !)^{m}}{(-1)^{m}} \sum_{k=1}^{\infty} \frac{(-1)^{m k} x^{k+n}}{\left[(k)_{n+1}\right]^{m}} \\
& +\sum_{k=0}^{n} x^{k}\left(\begin{array}{l}
n \\
k
\end{array}\right)^{m} Y_{m}\left[m B_{1}, m B_{2}, \ldots, m B_{m}\right]
\end{aligned}
$$

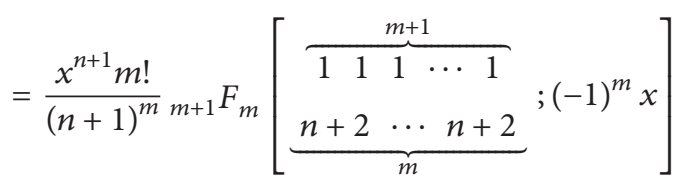

$$
\begin{aligned}
& +\sum_{k=0}^{n} x^{k}\left(\begin{array}{l}
n \\
k
\end{array}\right)^{m} Y_{m}\left[m B_{1}, m B_{2}, \ldots, m B_{m}\right] \text {. }
\end{aligned}
$$

Proof. Let us take $f(k, y)=1$ as a constant function in Theorem 1:

$$
\begin{aligned}
\sum_{k=1}^{\infty} & \frac{(-1)^{m k} x^{k+n}}{\left[(k)_{n+1}\right]^{m}} \\
= & \frac{(-1)^{m}}{m !(n !)^{m}} F_{m}^{(m)}(n, x) \\
& \quad-\sum_{k=0}^{n} x^{k}\left(\begin{array}{l}
n \\
k
\end{array}\right)^{m} Y_{m}\left[m B_{1}, m B_{2}, \ldots, m B_{m}\right] .
\end{aligned}
$$


It proves the first part of Theorem 3. Writing the function $F_{m}^{(m)}(n, x)$ and $F_{m}(n, x)$ as Hyper geometric functions defined in first section we can derive

$$
\begin{aligned}
& \frac{m !(n !)^{m}}{(-1)^{m}} \sum_{k=1}^{\infty} \frac{(-1)^{m k} x^{k+n}}{\left[(k)_{n+1}\right]^{m}}
\end{aligned}
$$

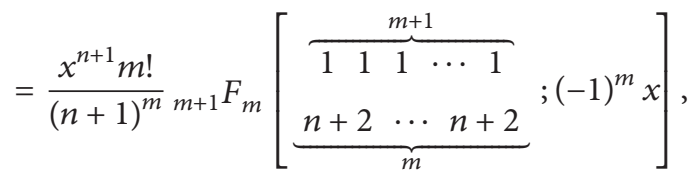

$$
\begin{aligned}
& F_{m}(r, x)=\sum_{k=0}^{\infty} x^{k}\left(\begin{array}{l}
r \\
k
\end{array}\right)^{m}
\end{aligned}
$$

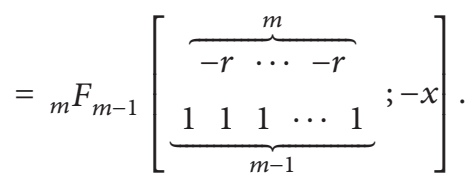

Finally combining (37) and (38) we can easily obtain Theorem 3.

Theorem 4. For integers $n \geqslant 0$ and $|x|<1$,

$$
\begin{aligned}
& \sum_{k=1}^{\infty} \frac{x^{k+n}}{(k)_{n+1}} H_{n+k}^{(1)}=\sum_{k=1}^{n} \frac{x^{k}}{n !}\left(\begin{array}{l}
n \\
k
\end{array}\right) H_{k}^{(1)}\left(H_{n}^{(1)}-H_{n-k}^{(1)}\right) \\
& \quad+\frac{1}{(1+x) n !} \Phi\left(\frac{1}{1+x}, 2, n+1\right) \\
& \quad-\frac{(1+x)^{n}}{n !}\left[H_{n}^{(1)} \ln (1+x)\right. \\
& \left.+\ln (1+x) \ln \left(\frac{x}{1+x}\right)+\zeta(2, n+1)\right] .
\end{aligned}
$$

Proof. Considering Theorem 2 for $m=1, y=1, f(k, y)=$ $H_{k}^{(1)}$ and using the property of bell polynomials,

$$
\begin{gathered}
\sum_{k=1}^{\infty} \frac{x^{k+n}}{(k)_{n+1}} H_{n+k}^{(1)}=\frac{-1}{n !}\left[\left.\frac{d}{d r} Q_{1}(r, x, 1)\right|_{r=n}\right. \\
\left.-\sum_{k=0}^{n} x^{k}\left(\begin{array}{l}
n \\
k
\end{array}\right) H_{k}^{(1)}\left(H_{n}^{(1)}-H_{n-k}^{(1)}\right)\right] .
\end{gathered}
$$

Taking into account an identity derived in Boyadzhiev's paper [8] titled "Harmonic Number Identities via Euler's Transform,"

$$
\begin{aligned}
Q_{1}(r, x, 1)= & H_{r}^{(1)}(1+x)^{r}-\sum_{k=1}^{r} \frac{(1+x)^{r-k}}{k} \\
= & H_{r}^{(1)}(1+x)^{r}+(1+x)^{r} \ln \left(\frac{x}{1+x}\right) \\
& -\sum_{k=1}^{\infty} \frac{(1+x)^{-k}}{k+r} .
\end{aligned}
$$

Differentiating the identity in (41) with respect to $r$ and using the known formula $\left.(d / d r) H_{r}^{(1)}\right|_{r=n}=\zeta(2, n+1)$,

$$
\begin{aligned}
& \left.\frac{d}{d r} Q_{1}(r, x, 1)\right|_{r=n}=(1+x)^{n}\left[H_{n}^{(1)} \ln (1+x)\right. \\
& \left.+\ln (1+x) \ln \left(\frac{x}{1+x}\right)+\zeta(2, n+1)\right] \\
& \quad-\sum_{k=0}^{\infty} \frac{(1+x)^{-k-1}}{(k+n+1)^{2}}=(1+x)^{n}\left[H_{n}^{(1)} \ln (1+x)\right. \\
& \left.+\ln (1+x) \ln \left(\frac{x}{1+x}\right)+\zeta(2, n+1)\right]-\frac{1}{(1+x)} \\
& \quad . \Phi\left(\frac{1}{1+x}, 2, n+1\right) .
\end{aligned}
$$

Considering the above expression together with (40) we can conclude Theorem 2. Special case of Theorem 2 for $x=1$ can be found in [5]. Theorem 2 also generalizes many identities derived in [9]. The finite summation in the right-hand side of Theorem 2 can be obtained from [7, 10] for different values of $x$. Interested readers can also find some computer assisted proofs of these identities in [11].

Theorem 5. For integers $n \geq 0$,

$$
\begin{aligned}
& \sum_{k=1}^{\infty} \frac{(-1)^{k}}{\left[(k)_{n+1}\right]^{2}} H_{n+k}^{(1)}=\frac{1}{(n !)^{2}} \sum_{k=1}^{n}\left(\begin{array}{l}
n \\
k
\end{array}\right)^{2} \\
& \cdot H_{k}^{(1)}\left[\left(H_{n}^{(2)}-H_{n-k}^{(2)}\right)-2\left(H_{n}^{(1)}-H_{n-k}^{(1)}\right)^{2}\right] \\
& +\frac{1}{(n !)^{2}}\left(\begin{array}{c}
2 n \\
n
\end{array}\right)\left[4\left(H_{2 n}^{(1)}-H_{n}^{(1)}\right)\left(H_{2 n}^{(2)}-H_{n}^{(2)}\right)\right. \\
& \left.+2 H_{2 n}^{(3)}-3 H_{2 n}^{(3)}+\zeta(3)\right]+\frac{1}{(n !)^{2}} \\
& \cdot\left(\begin{array}{c}
2 n \\
n
\end{array}\right)\left[\left(2 H_{n}^{(1)}-H_{2 n}^{(1)}\right)\right. \\
& \left.\cdot\left\{2\left(H_{2 n}^{(1)}-H_{n}^{(1)}\right)^{2}+\left(H_{n}^{(1)}-H_{2 n}^{(1)}\right)+\zeta(2)\right\}\right] .
\end{aligned}
$$

Proof. Setting $m=2, y=2, x=1$, and $f(k, y)=H_{k}^{(2)}$ in Theorem 2 and by virtue of Bell polynomials

$$
\begin{aligned}
& \sum_{k=1}^{\infty} \frac{(-1)^{k}}{\left[(k)_{n+1}\right]^{2}} H_{n+k}^{(1)}=\frac{1}{2(n !)^{2}}\left[\left.\frac{d^{2}}{d r^{2}} Q_{2}(r, 1,1)\right|_{r=n}\right. \\
& +\sum_{k=1}^{n}\left(\begin{array}{l}
n \\
k
\end{array}\right)^{2} \\
& \left.\cdot H_{k}^{(1)}\left[2\left(H_{n}^{(2)}-H_{n-k}^{(2)}\right)-4\left(H_{n}^{(1)}-H_{n-k}^{(1)}\right)^{2}\right]\right] .
\end{aligned}
$$


The following harmonic number identity can be found in many texts of mathematical literatures. Mainly Chu and De Donno [12] and Paule and Schneider [11] discussed these types of summation formulas in great detail.

$$
\mathrm{Q}_{2}(r, 1,1)=\sum_{k=1}^{r}\left(\begin{array}{l}
r \\
k
\end{array}\right)^{2} H_{k}^{(1)}=\left(\begin{array}{c}
2 r \\
r
\end{array}\right)\left(2 H_{r}^{(1)}-H_{2 r}^{(1)}\right) .
$$

Differentiating (45) two times with respect to $r$ and using the formula involving differentiation of generalized harmonic numbers stated in the first section,

$$
\begin{aligned}
& \left.\frac{d^{2}}{d r^{2}} Q_{2}(r, 1,1)\right|_{r=n} \\
& =\left.\frac{d^{2}}{d r^{2}}\left[\left(\begin{array}{c}
2 r \\
r
\end{array}\right)\left(2 H_{r}^{(1)}-H_{2 r}^{(1)}\right)\right]\right|_{r=n} \\
& =\left(\begin{array}{c}
2 n \\
n
\end{array}\right)\left[8\left(H_{2 n}^{(1)}-H_{n}^{(1)}\right)\left(H_{2 n}^{(2)}-H_{n}^{(2)}\right)+4 H_{2 n}^{(3)}\right. \\
& \left.-6 H_{2 n}^{(3)}+2 \zeta(3)\right]+\left(\begin{array}{c}
2 n \\
n
\end{array}\right)\left[2\left(2 H_{n}^{(1)}-H_{2 n}^{(1)}\right)\right. \\
& \left.\cdot\left\{2\left(H_{2 n}^{(1)}-H_{n}^{(1)}\right)^{2}+\left(H_{n}^{(1)}-H_{2 n}^{(1)}\right)+\zeta(2)\right\}\right] .
\end{aligned}
$$

Finally substituting the expressions of (45) and (46) in (44), we easily obtain Theorem 5 .

\section{Some Special Corollaries}

Corollary 6. For integers $n \geq 0, p \leq n$,

$$
\begin{aligned}
& \frac{x^{n+1}}{n+1}\left(\begin{array}{c}
n+1 \\
p
\end{array}\right){ }_{2} F_{1}\left[\begin{array}{c}
1 \\
1 \\
n-p+2
\end{array} ;-x\right] \\
& =\sum_{k=1}^{n} x^{k}\left(\begin{array}{l}
n \\
k
\end{array}\right)\left(\begin{array}{l}
k \\
p
\end{array}\right)\left(H_{n-k}^{(1)}-H_{n}^{(1)}\right)+x^{p}(1+x)^{n-p} \\
& \cdot\left(\begin{array}{l}
n \\
p
\end{array}\right)\left\{\ln (1+x)+\left(H_{n}^{(1)}-H_{n-p}^{(1)}\right)\right\} .
\end{aligned}
$$

Using the following identity (48) given in Volume 2 of Gould's book [13] and considering Theorem 2 for $m=1 ; f(k, p)=\left(\begin{array}{l}k \\ p\end{array}\right)$ and

$$
\sum_{k=0}^{n} x^{k}\left(\begin{array}{l}
n \\
k
\end{array}\right)\left(\begin{array}{l}
k \\
p
\end{array}\right)=x^{p}(1+x)^{n-p}\left(\begin{array}{l}
n \\
p
\end{array}\right)
$$

Some special cases of this formula can be found in the published literature [14-16].

Corollary 7. For integers $n \geq 0, p \geq 1$,

$$
\begin{aligned}
& \sum_{i=0}^{p-1}(-z)^{i}\left(\begin{array}{c}
n+p+1 \\
i
\end{array}\right){ }_{2} F_{1}\left[\begin{array}{c}
1 \\
n+p-i+2
\end{array} ; 1\right]=(1 \\
& -z)^{n-p+1}{ }_{2} F_{1}\left[\begin{array}{cc}
1 & 1 \\
n+p+2
\end{array} ; z\right]+(n+p+1) \\
& \cdot(-z)^{n+p}\left\{\ln (1+x)+\left(H_{n}^{(1)}-H_{n+p}^{(1)}\right)\right\}-\sum_{k=0}^{n}(-1)^{k} \\
& \cdot \frac{\left(\begin{array}{l}
n \\
k
\end{array}\right)\left(\begin{array}{c}
n+p+1 \\
p
\end{array}\right)}{\left(\begin{array}{c}
k+p \\
p
\end{array}\right)}\left(H_{n}^{(1)}-H_{n-k}^{(1)}\right) \\
& \left\{(1-z)^{k+p}-\sum_{i=0}^{p-1}(-z)^{i}\left(\begin{array}{c}
k+p \\
i
\end{array}\right)\right\}
\end{aligned}
$$

Considering the following identity (50) illustrated in Volume 2 of Gould's book [13] and using Theorem 2 for $p \geq 1 x=-1, m=1 ; f(k, p, z)=$ $\left(1 /\left(\begin{array}{c}k+p \\ k\end{array}\right)\right)\left[(1-z)^{k+p}-\sum_{i=0}^{p-1}(-z)^{i}\left(\begin{array}{c}k+p \\ i\end{array}\right)\right]$,

$$
\sum_{k=1}^{n}(-1)^{k}\left(\begin{array}{l}
n \\
k
\end{array}\right) f(k, p, z)=\frac{(-1)^{p}}{\left(\begin{array}{c}
n+p \\
p
\end{array}\right)} z^{n+p} .
$$

Corollary 8. For integers $n \geq 0$ and $p \geq 0$,

$$
\begin{aligned}
& \frac{(-1)^{n}(n+1)^{p+1}}{\prod_{i=1}^{\alpha} Y_{i}}{ }_{\alpha+p+1} F_{\alpha+p}\left[\begin{array}{ccc}
1 & \begin{array}{c}
\overbrace{n+2 \cdots n+2}^{p-1} \\
\underbrace{\frac{n+1 \cdots n+1}{Y_{1} \cdots Y_{\alpha}}}_{p}
\end{array} \underbrace{Y_{1}+1 \cdots Y_{\alpha}+1}_{\alpha}
\end{array}\right]=\sum_{j=1}^{\alpha} \frac{\left(X_{j}\right)^{p-1}\left(H_{n}^{(1)}-H_{n-X_{j}}^{(1)}\right)}{n !\left(\begin{array}{c}
n-X_{j} \\
n
\end{array}\right) \prod_{i=1, i \neq j}^{\alpha}\left(X_{j}-X_{i}\right)} \\
& \quad+\sum_{k=1}^{n} \frac{(-1)^{k}(k)^{p}\left(\begin{array}{l}
n \\
k
\end{array}\right)}{n ! \prod_{i=1}^{\alpha}\left(k-X_{i}\right)}\left(H_{n}^{(1)}-H_{n-k}^{(1)}\right) .
\end{aligned}
$$


Proof. The following identity is given in Volume 5 of Gould's book [13]:

$$
\sum_{k=1}^{n} \frac{(-1)^{k}(k)^{p}\left(\begin{array}{l}
n \\
k
\end{array}\right)}{n ! \prod_{i=1}^{\alpha}\left(k-X_{i}\right)}=-\sum_{j=1}^{\alpha} \frac{\left(X_{j}\right)^{p-1}}{\left(\begin{array}{c}
n-X_{j} \\
n
\end{array}\right) \prod_{i=1, i \neq j}^{\alpha}\left(X_{j}-X_{i}\right)} .
$$

Considering Theorem 2 for $x=-1, m=1 ; f\left(k, X_{i}\right)=$ $k^{p} / \prod_{i=1}^{\alpha}\left(k-X_{i}\right)$ and also using (52) we can immediately find Corollary 8 .

Corollary 9. For integers $n \geq 0, b \geq c, b$, and $c$ are positive integers,

$$
\begin{aligned}
& { }_{3} F_{2}\left[\begin{array}{ccc}
1 & 1 & n+b-c+2 \\
n+2 & n+b+2
\end{array} ; 1\right]=\frac{(-1)^{n}(n+1)}{\left(\begin{array}{c}
n+b+1 \\
c
\end{array}\right)} \\
& \cdot \sum_{k=1}^{n} \frac{(-1)^{k}\left(\begin{array}{l}
n \\
k
\end{array}\right)}{\left(\begin{array}{c}
k+b \\
c
\end{array}\right)}\left(H_{n}^{(1)}-H_{n-k}^{(1)}\right) \\
& -\frac{(-1)^{n}(n+1)}{\left(\begin{array}{c}
n+b+1 \\
c
\end{array}\right)}\left[\frac{-c}{(n+c)^{2}\left(\begin{array}{c}
n+b \\
b-c
\end{array}\right)}\right. \\
& \left.+\frac{c}{(n+c)\left(\begin{array}{c}
n+b \\
b-c
\end{array}\right)}\left(H_{n-c}^{(1)}-H_{n+b}^{(1)}\right)\right] \text {. }
\end{aligned}
$$

Using the following identity (54) given in Volume 5 of Gould's book [13] and considering Theorem 2 for $x=-1, m=$ $1 ; f(k, b, c)=1 /\left(\begin{array}{c}k+b \\ c\end{array}\right)$

$$
\sum_{k=1}^{n} \frac{(-1)^{k}\left(\begin{array}{l}
n \\
k
\end{array}\right)}{\left(\begin{array}{c}
k+b \\
c
\end{array}\right)}=\frac{c}{(n+c)\left(\begin{array}{c}
n+b \\
b-c
\end{array}\right)} \text {. }
$$

Terminating version of these kinds of hypergeometric series goes back to Bailey [15].

Differentiation of Laguerre Polynomials with respect to Its Order. Let $L_{n}(x)$ be the Laguerre Polynomials defined by

$$
L_{n}(x)=\sum_{k=1}^{n}(-1)^{k}\left(\begin{array}{l}
n \\
k
\end{array}\right) \frac{x^{k}}{k !} .
$$

Corollary 10. For integers $n \geq 0$,

$$
\begin{aligned}
& \left.\frac{d}{d r} L_{r}(x)\right|_{r=n} \\
& =\frac{(-x)^{n+1}}{(n+1)(n+1) !}{ }_{2} F_{2}\left[\begin{array}{cc}
1 & 1 \\
n+2 & n+2
\end{array} ; x\right] \\
& \quad+\sum_{k=1}^{n} \frac{(-x)^{k}}{k !}\left(\begin{array}{l}
n \\
k
\end{array}\right)\left(H_{n}^{(1)}-H_{n-k}^{(1)}\right) .
\end{aligned}
$$

Using the previous identity (55) for Laguerre Polynomials and considering Theorem 2 for $x=-x, m=1 ; f(k)=1 / k$ ! we can easily derive Corollary 10.
Corollary 11. For integers $m \geq 1, n \geq 0$, and $p=m+n$,

$$
\begin{aligned}
& { }_{4} F_{3}\left[\begin{array}{cccc}
1 & 1 & p & p \\
n+2 & p+1 & p+1
\end{array} ; 1\right]=(-1)^{n+1}(n+1)(m \\
& +n)^{2}\left[\frac{1}{m\left(\begin{array}{c}
p \\
n
\end{array}\right)}\left(H_{p}^{(1)}-H_{m-1}^{(1)}\right)\left(H_{n}^{(1)}-H_{p}^{(1)}\right)+\zeta(2)\right. \\
& \left.-H_{p}^{(2)}\right]+(-1)^{n}(n+1)(m+n)^{2} \\
& \cdot \sum_{k=1}^{n} \frac{(-1)^{k}}{(k+m)^{2}}\left(\begin{array}{l}
n \\
k
\end{array}\right)\left(H_{n}^{(1)}-H_{n-k}^{(1)}\right) \text {. }
\end{aligned}
$$

Making use of the following identity (58) derived in [17] and considering Theorem 2 for $x=-1, m=1 ; f(k, m)=1 /(k+$ $m)^{2}$,

$$
\sum_{k=1}^{n} \frac{(-1)^{k}}{(k+m)^{2}}\left(\begin{array}{l}
n \\
k
\end{array}\right)=\frac{1}{m\left(\begin{array}{c}
m+n \\
n
\end{array}\right)}\left(H_{m+n}^{(1)}-H_{m-1}^{(1)}\right) .
$$

Corollary 12. For integers $m \geq 1, n \geq 0$, and $p=m+n$,

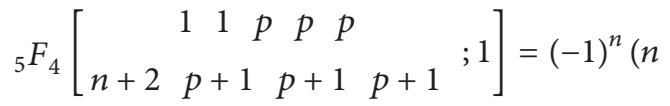

$$
\begin{aligned}
& \text { +1) }(m+n)^{3} \sum_{k=1}^{n} \frac{(-1)^{k}}{(k+m)^{3}}\left(\begin{array}{l}
n \\
k
\end{array}\right)\left(H_{n}^{(1)}-H_{n-k}^{(1)}\right) \\
& -\frac{(-1)^{n}(n+1) p^{3}}{m\left(\begin{array}{c}
p \\
n
\end{array}\right)}\left\{\zeta(2, p+1)\left(H_{p}^{(1)}-H_{m-1}^{(1)}\right)\right. \\
& +\zeta(3, p+1)\}-\frac{(-1)^{n}(n+1) p^{3}}{2 m\left(\begin{array}{c}
p \\
n
\end{array}\right)}\left(H_{n}^{(1)}-H_{p}^{(1)}\right) \\
& \cdot\left\{\left(H_{p}^{(1)}-H_{m-1}^{(1)}\right)^{2}+\left(H_{p}^{(2)}-H_{m-1}^{(2)}\right)\right\} \cdot
\end{aligned}
$$

Making use of the following identity (60) derived in [17] and considering Theorem 2 for $x=-1, m=1 ; f(k, m)=1 /(k+$ $m)^{3}$,

$$
\begin{aligned}
& \sum_{k=1}^{n} \frac{(-1)^{k}}{(k+m)^{3}}\left(\begin{array}{l}
n \\
k
\end{array}\right) \\
& =\frac{1}{2 m\left(\begin{array}{c}
m+n \\
n
\end{array}\right)}\left[\left(H_{m+n}^{(1)}-H_{m-1}^{(1)}\right)^{2}+\left(H_{m+n}^{(2)}-H_{m-1}^{(2)}\right)\right] .
\end{aligned}
$$


Corollary 13. For $x>-1$ and integers $n, p \geq 0$,

$$
\begin{aligned}
& { }_{p+2} F_{p+1}\left[\begin{array}{ccc}
1 & 1 & \underbrace{n-1}_{p+2 n+2 n+2 \cdots n+2}
\end{array} ;-x\right] \\
& =\frac{(-1)^{n+1}}{(n+1)^{p-1}} \sum_{k=0}^{n} x^{k}\left(\begin{array}{l}
n \\
k
\end{array}\right) k^{p}\left(H_{n}^{(1)}-H_{n-k}^{(1)}\right)+\frac{(-1)^{n}}{(n+1)^{p-1}} \\
& \cdot \sum_{j=0}^{p} x^{j}(1+x)^{n-j}\left(\begin{array}{l}
n \\
j
\end{array}\right) j ! S(p, j) \\
& \cdot\left[\ln (1+x)+\left(H_{n}^{(1)}-H_{n-j}^{(1)}\right)\right],
\end{aligned}
$$

where $S(p, j)$ are Stirling numbers of the second kind. Considering the following identity (62) given in [8] for $x=y$ and applying Theorem 2 for, $x=1, m=1 ; f(k, p)=k^{p}$,

$$
\sum_{k=0}^{n} x^{k}\left(\begin{array}{l}
n \\
k
\end{array}\right) k^{p}=\sum_{j=0}^{p} x^{j}(1+x)^{n-j}\left(\begin{array}{l}
n \\
j
\end{array}\right) j ! S(p, j) .
$$

Corollary 14. For $|x|<1$ and integers $n \geq 0$,

$$
\begin{aligned}
& \frac{x^{n+1}}{n+1}{ }_{2} F_{1}\left[\begin{array}{cc}
1 & 1 \\
n+2
\end{array} ; x\right] \\
& \quad=(-1)^{n+1}(1-x)^{n} \ln (1+x) \\
& \quad+(-1)^{n} \sum_{k=0}^{n}(-x)^{k}\left(\begin{array}{l}
n \\
k
\end{array}\right)\left(H_{n}^{(1)}-H_{n-k}^{(1)}\right) .
\end{aligned}
$$

Proof. Substituting $m=1$ in Theorem 3,

$$
\begin{aligned}
& \sum_{k=1}^{\infty} \frac{(-1)^{k} x^{k+n}}{(k)_{n+1}} \\
& \quad=-\frac{1}{n !}\left[F_{1}^{(1)}(n, x)-\sum_{k=0}^{n} x^{k}\left(\begin{array}{l}
n \\
k
\end{array}\right)\left(H_{n}^{(1)}-H_{n-k}^{(1)}\right)\right] .
\end{aligned}
$$

Using Newton's binomial theorem we have

$$
F_{1}(r, x)=\sum_{k=0}^{r} x^{k}\left(\begin{array}{l}
r \\
k
\end{array}\right)
$$

This implies

$$
\begin{aligned}
F_{1}^{(1)}(n, x) & =\left.\frac{d}{d r} F_{1}(r, x)\right|_{r=n}=\left.\frac{d}{d r}(1+x)^{r}\right|_{r=n} \\
& =(1+x)^{n} \ln (1+x) .
\end{aligned}
$$

Substituting the value of $F_{1}^{(1)}(n, x)$ from (66) in (64) and after some modifications we can obtain Corollary 14 . The modified version of Corollary 14 can be found in [18]. Zeilberger proved quite interesting properties of these types of hypergeometric functions in [19].
Corollary 15. For integers $n \geq 0$,

$$
\begin{aligned}
& \frac{1}{(n+1)^{2}}{ }_{3} F_{2}\left[\begin{array}{ccc}
1 & 1 & 1 \\
n+2 & n+2
\end{array} ; 1\right] \\
& =\left(\begin{array}{c}
2 n \\
n
\end{array}\right)\left[2\left(H_{2 n}^{(1)}-H_{n}^{(1)}\right)^{2}+H_{n}^{(2)}-2 H_{2 n}^{(2)}+\zeta(2)\right] \\
& \quad-\sum_{k=0}^{n}\left(\begin{array}{l}
n \\
k
\end{array}\right)^{2}\left[2\left(H_{n}^{(1)}-H_{n-k}^{(1)}\right)^{2}-\left(H_{n}^{(2)}-H_{n-k}^{(2)}\right)\right] .
\end{aligned}
$$

Proof. Substituting $m=2$ and $x=1$ in Theorem 3 and using the property of Bell polynomials,

$$
\begin{gathered}
\sum_{k=1}^{\infty} \frac{1}{\left[(k)_{n+1}\right]^{2}}=-\frac{1}{2(n !)^{2}}\left[F_{2}^{(2)}(n, 1)-\sum_{k=0}^{n}\left(\begin{array}{l}
n \\
k
\end{array}\right)^{2}\right. \\
\left.\cdot\left[4\left(H_{n}^{(1)}-H_{n-k}^{(1)}\right)^{2}-2\left(H_{n}^{(2)}-H_{n-k}^{(2)}\right)\right]\right] .
\end{gathered}
$$

Another classical result, special case of the Vandermonde Convolution, is given by

$$
F_{2}(r, 1)=\sum_{k=0}^{r}\left(\begin{array}{l}
r \\
k
\end{array}\right)^{2}=\left(\begin{array}{c}
2 r \\
r
\end{array}\right) .
$$

Hence we have

$$
\begin{aligned}
F_{2}^{(2)} & (n, 1)=\left.\frac{d^{2}}{d r^{2}}\left(\begin{array}{c}
2 r \\
r
\end{array}\right)\right|_{r=n} \\
& =\left(\begin{array}{c}
2 n \\
n
\end{array}\right)\left[4\left(H_{2 n}^{(1)}-H_{n}^{(1)}\right)^{2}+2 H_{n}^{(2)}-4 H_{2 n}^{(2)}\right. \\
& +2 \zeta(2)] .
\end{aligned}
$$

Considering the value of $F_{2}^{(2)}(n, 1)$ from (70) in (68) we finally recover Corollary 15.

Corollary 16. For integers $n \geq 0$,

$$
\begin{aligned}
& \frac{1}{(n+1)^{2}}{ }_{3} F_{2}\left[\begin{array}{ccc}
1 & 1 & 1 \\
n+2 & n+2
\end{array} ;-1\right] \\
& =\frac{(-1)^{n} 2^{n-3} \sqrt{\pi}}{\Gamma((n+2) / 2) \Gamma((1-n) / 2)}\left[\zeta\left(2, \frac{1-n}{2}\right)\right. \\
& \left.+\zeta\left(2, \frac{n+2}{2}\right)\right]+\sum_{k=0}^{n}(-1)^{n+k}\left(\begin{array}{l}
n \\
k
\end{array}\right)^{2} \\
& +\left[2\left(H_{n}^{(1)}-H_{n-k}^{(1)}\right)^{2}-\left(H_{n}^{(2)}-H_{n-k}^{(2)}\right)\right] \\
& +\frac{(-1)^{n+1} 2^{n-1} \sqrt{\pi}}{\Gamma((n+2) / 2) \Gamma((1-n) / 2)}\left[(\ln 2)^{2}\right. \\
& \left.+\ln 2\left(H_{-(n+1) / 2}^{(1)}-H_{n / 2}^{(1)}\right)+\left(H_{-(n+1) / 2}^{(1)}-H_{n / 2}^{(1)}\right)^{2}\right] .
\end{aligned}
$$


Proof. Substituting $m=2$ and $x=-1$ in Theorem 3 and using the property of Bell polynomials,

$$
\begin{aligned}
\sum_{k=1}^{\infty} \frac{(-1)^{k+n}}{\left[(k)_{n+1}\right]^{2}}=-\frac{1}{2(n !)^{2}}\left[F_{2}^{(2)}(n,-1)\right. \\
\quad-\sum_{k=0}^{n}(-1)^{k}\left(\begin{array}{l}
n \\
k
\end{array}\right)^{2} \\
\left.\quad \cdot\left[4\left(H_{n}^{(1)}-H_{n-k}^{(1)}\right)^{2}-2\left(H_{n}^{(2)}-H_{n-k}^{(2)}\right)\right]\right]
\end{aligned}
$$

Considering another classical result illustrated in Gould's book [13] Volume 5 which first appeared in American Math Monthly, for the case $z=r$, we have

$$
\begin{aligned}
F_{2}(r,-1) & =\sum_{k=0}^{r}(-1)^{k}\left(\begin{array}{l}
r \\
k
\end{array}\right)^{2} \\
& =\frac{2^{r} \sqrt{\pi}}{\Gamma((r+2) / 2) \Gamma((1-r) / 2)} .
\end{aligned}
$$

Hence we have

$$
\begin{aligned}
& F_{2}^{(2)}(n,-1)=\left.\frac{d^{2}}{d r^{2}}\left\{\frac{2^{r} \sqrt{\pi}}{\Gamma((r+2) / 2) \Gamma((1-r) / 2)}\right\}\right|_{r=n} \\
& \quad=\frac{(-1)^{n} 2^{n-2} \sqrt{\pi}}{\Gamma((n+2) / 2) \Gamma((1-n) / 2)}\left[\zeta\left(2, \frac{1-n}{2}\right)\right. \\
& \left.+\zeta\left(2, \frac{n+2}{2}\right)\right] \\
& +\frac{2^{n} \sqrt{\pi}}{\Gamma((n+2) / 2) \Gamma((1-n) / 2)}\left[(\ln 2)^{2}\right. \\
& \left.+\ln 2\left(H_{-(n+1) / 2}^{(1)}-H_{n / 2}^{(1)}\right)+\left(H_{-(n+1) / 2}^{(1)}-H_{n / 2}^{(1)}\right)^{2}\right] .
\end{aligned}
$$

Substituting the value of $F_{2}^{(2)}(n,-1)$ from (74) in (72) we can immediately obtain Corollary 16 . Various special cases of Corollary 16 can be found in several works of Wimp $[20,21]$.

Corollary 17. For integers $n \geq 0$,

$$
\begin{aligned}
& \frac{1}{(n+1)^{3}}{ }_{4} F_{3}\left[\begin{array}{cccc}
1 & 1 & 1 & 1 \\
n+2 & n+2 & n+2
\end{array} ;-1\right]=\frac{(-1)^{n}}{6} \\
& \cdot F_{3}^{(3)}(n,-1)-\sum_{k=0}^{n} \frac{(-1)^{n+k}}{2}\left(\begin{array}{l}
n \\
k
\end{array}\right)^{3} \\
& \cdot\left[9\left(H_{n}^{(1)}-H_{n-k}^{(1)}\right)^{3}\right. \\
& -9\left(H_{n}^{(1)}-H_{n-k}^{(1)}\right)\left(H_{n}^{(2)}-H_{n-k}^{(2)}\right) \\
& \left.+2\left(H_{n}^{(3)}-H_{n-k}^{(3)}\right)\right] .
\end{aligned}
$$

Proof. Substituting $m=3$ and $x=-1$ in Theorem 3 and using the property of Bell polynomials,

$$
\begin{aligned}
\sum_{k=1}^{\infty} \frac{(-1)^{k+n}}{\left[(k)_{n+1}\right]^{3}}=-\frac{1}{3 !(n !)^{3}}\left[F_{3}^{(3)}(n,-1)\right. \\
\left.\quad-\sum_{k=0}^{n}(-1)^{k}\left(\begin{array}{l}
n \\
k
\end{array}\right)^{3} Y_{3}\left[m B_{1}, m B_{2}, m B_{3}\right]\right] .
\end{aligned}
$$

Considering another classical result illustrated in volume 5 of Gould's book [13] which first appeared in American Math Monthly, considering for the case $z=r$ that

$$
\begin{aligned}
F_{3}(r,-1)=\sum_{k=0}^{r}(-1)^{k}\left(\begin{array}{l}
r \\
k
\end{array}\right)^{3} \\
=\frac{\Gamma(r+1) \Gamma(-r) \sqrt{\pi}}{2 \Gamma^{3}((r+2) / 2) \Gamma(-3 r / 2)}\left[1+(-1)^{r}\right] \\
=\frac{\Gamma(r+1) \Gamma(-r)}{2 \Gamma^{3}((r+2) / 2) \Gamma(-3 r / 2)} \\
\quad+\frac{\sqrt{\pi} \Gamma^{2}(r+1) \Gamma(-r)}{2^{r+1} \Gamma^{4}((r+2) / 2) \Gamma(-3 r / 2) \Gamma((r-1) / 2)} \\
=y_{1}+y_{2},
\end{aligned}
$$

hence we have

$$
\begin{aligned}
\ln \left(y_{1}\right)= & \ln \Gamma(r+1)+\ln \Gamma(-r)-\ln 2 \\
& -3 \ln \Gamma\left(\frac{r+2}{2}\right)-\ln \Gamma\left(\frac{-3 r}{2}\right), \\
\ln \left(y_{2}\right)= & \frac{1}{2} \ln \pi+2 \ln \Gamma(r+1)+\ln \Gamma(-r) \\
& -(r+1) \ln 2-4 \ln \Gamma\left(\frac{r+2}{2}\right) \\
& -\ln \Gamma\left(\frac{-3 r}{2}\right)-\ln \Gamma\left(\frac{r-1}{2}\right) .
\end{aligned}
$$

Now differentiating the above identities with respect to $r$ and using the definition of polygamma function,

$$
\begin{aligned}
& \frac{d}{d r} y_{1}=y_{1}\left[\psi(r+1)-\psi(-r)-\frac{3}{2} \psi\left(\frac{r+2}{2}\right)\right. \\
& \left.\quad+\frac{3}{2} \psi\left(\frac{-3 r}{2}\right)\right]=y_{1} \theta_{0}(r), \\
& \frac{d}{d r} y_{2}=y_{2}\left[2 \psi(r+1)-\psi(-r)-\ln 2-2 \psi\left(\frac{r+2}{2}\right)\right. \\
& \left.\quad+\frac{3}{2} \psi\left(\frac{-3 r}{2}\right)-\frac{1}{2} \psi\left(\frac{r-1}{2}\right)\right]=y_{2} \rho_{0}(r) .
\end{aligned}
$$


We also define

$$
\begin{aligned}
\theta_{1}(r)= & \frac{d}{d r} \theta_{0}(r) \\
= & \psi^{(1)}(r+1)+\psi^{(1)}(-r)-\frac{3}{4} \psi^{(1)}\left(\frac{r+2}{2}\right) \\
& -\frac{9}{4} \psi^{(1)}\left(\frac{-3 r}{2}\right), \\
\theta_{2}(r)= & \frac{d^{2}}{d r^{2}} \theta_{0}(r) \\
= & \psi^{(2)}(r+1)-\psi^{(2)}(-r)-\frac{3}{8} \psi^{(2)}\left(\frac{r+2}{2}\right) \\
& +\frac{27}{8} \psi^{(2)}\left(\frac{-3 r}{2}\right), \\
\rho_{1}(r)= & \frac{d}{d r} \rho_{0}(r) \\
= & 2 \psi^{(1)}(r+1)+\psi^{(1)}(-r)-\psi^{(1)}\left(\frac{r+2}{2}\right) \\
& -\frac{1}{4} \psi^{(1)}\left(\frac{r-1}{2}\right)-\frac{9}{4} \psi^{(1)}\left(\frac{-3 r}{2}\right), \\
\rho_{2}(r)= & \frac{d^{2}}{d r^{2}} \rho_{0}(r) \\
= & 2 \psi^{(2)}(r+1)-\psi^{(1)}(-r)-\frac{1}{2} \psi^{(2)}\left(\frac{r+2}{2}\right) \\
& \\
&
\end{aligned}
$$

Finally we have

$$
\begin{aligned}
F_{3}^{(3)}(n,-1) & \\
= & y_{1}\left[\theta_{0}^{3}(n)+3 \theta_{0}(n) \theta_{1}(n)+\theta_{2}(n)\right] \\
& +y_{2}\left[\rho_{0}^{3}(n)+3 \rho_{0}(n) \rho_{1}(n)+\rho_{2}(n)\right], \\
Y_{3}[ & \left.m B_{1}, m B_{2}, m B_{3}\right] \\
= & 27\left(H_{n}^{(1)}-H_{n-k}^{(1)}\right)^{3} \\
& -27\left(H_{n}^{(1)}-H_{n-k}^{(1)}\right)\left(H_{n}^{(2)}-H_{n-k}^{(2)}\right) \\
& +6\left(H_{n}^{(3)}-H_{n-k}^{(3)}\right) .
\end{aligned}
$$

Compiling (76), (77), and (81) we can obtain Corollary 17.

Some $q$ Series Identities. $q$-Pochhammer symbol, also called $q$ shifted factorial, is a $q$-analog of the common Pochhammer symbol. It is defined as

$$
\begin{aligned}
& (a ; q)_{n}=(1-a)(1-a q) \cdots\left(1-a q^{n-1}\right), \\
& (q ; q)_{n}=(q)_{n}=(1-q)\left(1-q^{2}\right) \cdots\left(1-q^{n}\right) .
\end{aligned}
$$

And the $q$ binomial coefficients also known as Gaussian coefficients, Gaussian polynomials, or Gaussian binomial coefficients are

$$
\left[\begin{array}{l}
n \\
m
\end{array}\right]_{q}=\frac{(q)_{n}}{(q)_{n-k}(q)_{k}}
$$

Let us define

$$
\begin{aligned}
Q(q, n, m) & =\frac{d^{m}}{d n^{m}}\left[\ln (q)_{n}\right]^{m}, \\
H_{(a ; q)}^{(m)} & =\sum_{k=0}^{n-1} \frac{1}{\left(1-a q^{k}\right)^{m}} ; \\
S(q, m) & =\sum_{k=1}^{\infty} \frac{q^{k}}{\left(1-q^{k}\right)} k^{m-1}, \\
R(q, a) & =\sum_{k=1}^{\infty} \frac{a^{k}}{\left(1-q^{k}\right)} .
\end{aligned}
$$

The operator $q D$, used extensively in several references, was recently used by authors in $[3,8,22]$.

$$
\begin{aligned}
(q D)^{m} f(q) & =\sum_{k=0}^{m}\left\{\begin{array}{l}
m \\
k
\end{array}\right\} q^{k} f^{(k)}(q), \\
(q D)^{m} q^{k} & =k^{m} q^{k} .
\end{aligned}
$$

Now by the properties of Bell Polynomials we have

$$
\begin{aligned}
& \frac{d^{m}}{d n^{m}}\left[(q)_{n}\right]^{m}=\frac{d^{m}}{d n^{m}} e^{\left[\ln (q)_{n}\right]^{m}}=\left[(q)_{n}\right]^{m} \\
& \quad \cdot Y_{m}[m Q(q, n, 1), m Q(q, n, 2), \ldots, m Q(q, n, m)] .
\end{aligned}
$$

We can further deduce

$$
\begin{aligned}
& {\left[\ln (q)_{n}\right]^{m}=m \ln (q)_{n}} \\
& \quad=m\left[\ln (1-q)+\ln \left(1-q^{2}\right)+\cdots+\ln \left(1-q^{n}\right)\right] \\
& \quad=-m \sum_{k=1}^{\infty} \frac{q^{k}}{k}\left[1+q^{k}+\cdots+q^{k(n-1)}\right] \\
& \quad=-m \sum_{k=1}^{\infty} \frac{q^{k}\left(1-q^{n k}\right)}{k\left(1-q^{k}\right)} .
\end{aligned}
$$

This implies

$$
\begin{aligned}
\frac{d^{m}}{d n^{m}} & {\left[\ln (q)_{n}\right]^{m}=m(\ln q)^{m} \sum_{k=1}^{\infty} \frac{q^{k(n+1)} k^{m}}{k\left(1-q^{k}\right)} } \\
= & m(\ln q)^{m} S(q, m) \\
& \quad-m(\ln q)^{m} \sum_{k=1}^{\infty} k^{m-1} q^{k}\left[1+q^{k}+\cdots+q^{k(n-1)}\right] .
\end{aligned}
$$


From the geometric series we have

$$
\sum_{k=1}^{\infty} q^{k}=\frac{1}{1-q}-1
$$

Applying the operator $q D$ on both sides $m$ times,

$$
\sum_{k=1}^{\infty} k^{m} q^{k}=\left(\frac{1}{1-q}-1\right)+\sum_{k=1}^{m}\left\{\begin{array}{l}
m \\
k
\end{array}\right\} \frac{q^{k} k !}{(1-q)^{k+1}} .
$$

By means of this identity we have

$$
\begin{aligned}
& \sum_{k=1}^{\infty} k^{m-1}\left[q^{k}+\cdots+q^{n k}\right]=\sum_{v=1}^{n} \sum_{k=1}^{\infty} k^{m-1} q^{v k} \\
& =\left(H_{(q)_{n}}^{(1)}-n\right)+\sum_{v=1}^{n} \sum_{k=1}^{m-1}\left\{\begin{array}{c}
m-1 \\
k
\end{array}\right\} \frac{q^{v k} k !}{\left(1-q^{v}\right)^{k+1}} .
\end{aligned}
$$

Finally

$$
\begin{aligned}
\frac{d^{m}}{d n^{m}}\left[\ln (q)_{n}\right]^{m}=Q(q, n, m) & \\
= & m(\ln q)^{m} S(q, m) \\
& \quad-m(\ln q)^{m} \sum_{k=1}^{\infty} k^{m-1} q^{k}\left[1+q^{k}+\cdots+q^{k(n-1)}\right] \\
= & m(\ln q)^{m} S(q, m)-m(\ln q)^{m}\left(H_{(q)_{n}}^{(1)}-n\right) \\
& \quad-m(\ln q)^{m} \sum_{v=1}^{n} \sum_{k=1}^{m-1}\left\{\begin{array}{c}
m-1 \\
k
\end{array}\right\} \frac{q^{v k} k !}{\left(1-q^{v}\right)^{k+1}} .
\end{aligned}
$$

Now

$$
\begin{aligned}
Q(q, n, k, m)=\frac{d^{m}}{d n^{m}}\left\{\ln \left(\left[\begin{array}{l}
n \\
k
\end{array}\right]_{q}\right)^{m}\right\} \\
=\frac{d^{m}}{d n^{m}}\left\{\left[\ln (q)_{n}\right]^{m}-\left[\ln (q)_{k}\right]^{m}-\left[\ln (q)_{n-k}\right]^{m}\right\} \\
=m(\ln q)^{m}\left(H_{(q)_{n-k}}^{(1)}-H_{(q)_{n}}^{(1)}+k\right) \\
\quad-m(\ln q)^{m} \sum_{v=n-k+1}^{n} \sum_{u=1}^{m-1}\left\{\begin{array}{c}
m-1 \\
u
\end{array}\right\} \frac{q^{v u} u !}{\left(1-q^{v}\right)^{u+1}} \\
=m(\ln q)^{m}\left(H_{(q)_{n-k}}^{(1)}-H_{(q)_{n}}^{(1)}+k\right) \\
\quad-m(\ln q)^{m} \sum_{v=1}^{k} \sum_{u=1}^{m-1}\left\{\begin{array}{c}
m-1 \\
u
\end{array}\right\} \frac{q^{(n-k+v) u} u !}{\left(1-q^{n-k+v}\right)^{u+1}} .
\end{aligned}
$$

By virtue of Bell Polynomials we have

$$
\begin{aligned}
& \frac{d^{m}}{d n^{m}}\left(\left[\begin{array}{l}
n \\
k
\end{array}\right]_{q}\right)^{m}=\frac{d^{m}}{d n^{m}} e^{\ln \left(\left[\begin{array}{l}
n \\
k
\end{array}\right]_{q}\right)^{m}}=\left(\left[\begin{array}{l}
n \\
k
\end{array}\right]_{q}\right)^{m} \\
& \cdot Y_{m}[m Q(q, n, k, 1), m Q(q, n, k, 2), \ldots, \\
& m Q(q, n, k, m)] .
\end{aligned}
$$

Case 1. Substituting $m=1$ in (94),

$$
\frac{d}{d n}\left[\begin{array}{l}
n \\
k
\end{array}\right]_{q}=\left[\begin{array}{l}
n \\
k
\end{array}\right]_{q} \ln q\left[k+H_{(q)_{n-k}}^{(1)}-H_{(q)_{n}}^{(1)}\right] .
$$

And for $r$ is an integer,

$$
\left.\frac{d}{d n}\left[\begin{array}{l}
n \\
k
\end{array}\right]_{q}\right|_{n=r}=\left[\begin{array}{l}
r \\
k
\end{array}\right]_{q} \ln q\left[k+H_{(q)_{r-k}}^{(1)}-H_{(q)_{r}}^{(1)}\right] .
$$

Finally using the definition of (93) we can derive

$$
\begin{aligned}
& \lim _{k \rightarrow r+v} Q(q, r, k, 1) \\
& =\lim _{k \rightarrow r+v}\left\{\left[\begin{array}{l}
r \\
k
\end{array}\right]_{q} \ln q\left[k+H_{(q)_{r-k}}^{(1)}-H_{(q)_{r}}^{(1)}\right]\right\} \\
& =\ln q \frac{(-1)^{v}(q)_{r}(q)_{v-1}}{q^{v(v-1) / 2}(q)_{r+v}} .
\end{aligned}
$$

Case 2. Substituting $m=2$ in (94), we already derived the expression for $Q(q, r, k, 1)$.

$$
\begin{aligned}
& Q(q, r, k, 2) \\
& =2(\ln q)^{2}\left\{k+H_{(q)_{r-k}}^{(1)}-H_{(q)_{r}}^{(1)}-\sum_{v=1}^{k} \frac{q^{n-k+v}}{\left(1-q^{n-k+v}\right)^{2}}\right\} \\
& =2(\ln q)^{2}\left\{k+H_{(q)_{r-k}}^{(2)}-H_{(q)_{r}}^{(2)}\right\} .
\end{aligned}
$$

Finally using (94) for $m=2$ we have

$$
\begin{gathered}
\frac{d^{2}}{d n^{2}}\left(\left[\begin{array}{l}
n \\
k
\end{array}\right]_{q}\right)^{2}=\left(\left[\begin{array}{l}
n \\
k
\end{array}\right]_{q}\right)^{2} \\
\cdot Y_{2}[2 Q(q, n, k, 1), 2 Q(q, n, k, 2)]=\left(\left[\begin{array}{l}
n \\
k
\end{array}\right]_{q}\right)^{2} \\
\cdot\left\{4(Q(q, n, k, 1))^{2}+2 Q(q, n, k, 2)\right\}=4(\ln q)^{2} \\
\cdot\left(\left[\begin{array}{l}
n \\
k
\end{array}\right]_{q}\right)^{2} \\
\cdot\left[\left\{k+H_{(q)_{n-k}}^{(1)}-H_{(q)_{n}}^{(1)}\right\}^{2}+\left\{k+H_{(q)_{n-k}}^{(2)}-H_{(q)_{n}}^{(2)}\right\}\right] .
\end{gathered}
$$

From previous calculation discussed in (97) we have

$$
\begin{gathered}
\lim _{k \rightarrow r+v}\left\{\left[\begin{array}{l}
r \\
k
\end{array}\right]_{q}\left[k+H_{(q)_{r-k}}^{(1)}-H_{(q)_{r}}^{(1)}\right]\right\}^{2} \\
=\frac{(q)_{r}^{2}(q)_{v-1}^{2}}{q^{v(v-1)}(q)_{r+v}^{2}} .
\end{gathered}
$$


By the same procedure we have

$$
\lim _{k \rightarrow r+v}\left[\begin{array}{l}
r \\
k
\end{array}\right]_{q}^{2}\left[k+H_{(q)_{r-k}}^{(2)}-H_{(q)_{r}}^{(2)}\right]=-\frac{(q)_{r}^{2}(q)_{v-1}^{2}}{q^{v(v-1)}(q)_{r+v}^{2}}
$$

So finally

$$
\begin{aligned}
& \lim _{k \rightarrow r+v} 4(\ln q)^{2}\left(\left[\begin{array}{l}
n \\
k
\end{array}\right]_{q}\right)^{2} \\
& \quad \cdot\left[\left\{k+H_{(q)_{n-k}}^{(1)}-H_{(q)_{n}}^{(1)}\right\}^{2}+\left\{k+H_{(q)_{n-k}}^{(2)}-H_{(q)_{n}}^{(2)}\right\}\right] \\
& \quad=0 .
\end{aligned}
$$

\section{Theorems Closely Related to Roger-Ramanujan Identities}

Roger-Ramanujan Identities. Rogers Ramanujan identities are given in

$$
\begin{aligned}
& \sum_{k=0}^{\infty} \frac{q^{k^{2}}}{(q ; q)_{k}}=\frac{1}{\left(q ; q^{5}\right)_{\infty}\left(q^{4} ; q^{5}\right)_{\infty}} \\
& \sum_{k=0}^{\infty} \frac{q^{k(k+1)}}{(q ; q)_{k}}=\frac{1}{\left(q^{2} ; q^{5}\right)_{\infty}\left(q^{3} ; q^{5}\right)_{\infty}}
\end{aligned}
$$

Roger discovered these identities in 1894 [23, 24], but they were entirely ignored until Ramanujan rediscovered them about 20 years later. A detailed history of these identities can be found in great detail in the survey article written by Andrews [25].

Theorem 18. One has

$$
\begin{aligned}
\sum_{v=1}^{\infty} \frac{(q)_{v-1}}{(q)_{r+v}}\left(a q^{r}\right)^{v} & \\
= & \frac{(a ; q)_{r}}{(-a)^{r}(q)_{r} q^{r(r-1) / 2}}\left[R(q, a)+r-H_{(a ; q)_{r}}^{(1)}\right] \\
& \quad-\sum_{k=0}^{r} \frac{(-a)^{k-r} q^{(k-r)(k+r-1) / 2}}{(q)_{k}(q)_{r-k}}\left[k+H_{(q)_{r-k}}^{(1)}-H_{(q)_{r}}^{(1)}\right] .
\end{aligned}
$$

Proof. From the $q$ binomial theorem,

$$
(a ; q)_{n}=\sum_{k=0}^{\infty}\left[\begin{array}{l}
n \\
k
\end{array}\right]_{q}(-a)^{k} q^{k(k-1) / 2} .
$$

Differentiating both sides of (105) with respect to $n$ at the point $n=r$ (integer),

$$
\begin{aligned}
& \left.\frac{d}{d n}(a ; q)_{n}\right|_{n=r}=\ln q(a ; q)_{r} \sum_{v=1}^{\infty} \frac{\left(a q^{r}\right)^{v}}{1-q^{v}} \\
& =\ln q \sum_{k=0}^{\infty}(-a)^{k} q^{k(k-1) / 2}\left[\begin{array}{l}
r \\
k
\end{array}\right]_{q}\left[k+H_{(q)_{r-k}}^{(1)}-H_{(q)_{r}}^{(1)}\right] \\
& =\ln q \sum_{k=0}^{r}(-a)^{k} q^{k(k-1) / 2}\left[\begin{array}{l}
r \\
k
\end{array}\right]_{q}\left[k+H_{(q)_{r-k}}^{(1)}-H_{(q)_{r}}^{(1)}\right] \\
& +\ln q(-a)^{r}(q)_{r} q^{r(r-1) / 2} \sum_{v=1}^{\infty} \frac{(q)_{v-1}}{(q)_{r+v}}\left(a q^{r}\right)^{v} .
\end{aligned}
$$

After some calculations we have Theorem 18:

$$
\begin{aligned}
\sum_{v=1}^{\infty} \frac{(q)_{v-1}}{(q)_{r+v}}\left(a q^{r}\right)^{v} & \\
= & \frac{(a ; q)_{r}}{(-a)^{r}(q)_{r} q^{r(r-1) / 2}}\left[R(q, a)+r-H_{(a ; q)_{r}}^{(1)}\right] \\
& \quad-\sum_{k=0}^{r} \frac{(-a)^{k-r} q^{(k-r)(k+r-1) / 2}}{(q)_{k}(q)_{r-k}}\left[k+H_{(q)_{r-k}}^{(1)}-H_{(q)_{r}}^{(1)}\right] .
\end{aligned}
$$

Theorem 19. One has

$$
\begin{aligned}
\sum_{k=0}^{n} q^{k^{2}}\left[\begin{array}{l}
r \\
k
\end{array}\right]_{q}^{2} & \\
\cdot & {\left[\left\{k+H_{(q)_{r-k}}^{(1)}-H_{(q)_{r}}^{(1)}\right\}^{2}+\left\{k+H_{(q)_{r-k}}^{(2)}-H_{(q)_{r}}^{(2)}\right\}\right] } \\
= & {\left[\begin{array}{c}
2 r \\
r
\end{array}\right]_{q}\left[\left\{r+H_{(q)_{r}}^{(1)}-H_{(q)_{2 r}}^{(1)}\right\}^{2}+\frac{1}{2} S(q, 2)\right.} \\
+ & \left.H_{(q)_{2 r}}^{(1)}-H_{(q)_{2 r}}^{(2)}+\frac{1}{2}\left(H_{(q)_{r}}^{(2)}-H_{(q)_{r}}^{(1)}\right)\right] .
\end{aligned}
$$

Proof. The proof is similar to the previous one. For this case we have to consider the identity

$$
\sum_{k=0}^{\infty} q^{k^{2}}\left[\begin{array}{l}
n \\
k
\end{array}\right]_{q}^{2}=\left[\begin{array}{l}
2 n \\
n
\end{array}\right]_{q}
$$

Differentiating (109) with respect to $n$ two times when $n=r$ (integer) and considering (99) and (102) together we have,

$$
\begin{aligned}
& \sum_{k=0}^{r} q^{k^{2}}\left[\begin{array}{l}
r \\
k
\end{array}\right]_{q}^{2} \\
& \cdot\left[\left\{k+H_{(q)_{r-k}}^{(1)}-H_{(q)_{r}}^{(1)}\right\}^{2}+\left\{k+H_{(q)_{r-k}}^{(2)}-H_{(q)_{r}}^{(2)}\right\}\right] \\
& \quad=\frac{d^{2}}{d n^{2}}\left[\begin{array}{c}
2 n \\
n
\end{array}\right]_{q} .
\end{aligned}
$$


Using the same idea stated above we can calculate

$$
\begin{aligned}
& \left.\frac{d}{d n} \ln \left[\begin{array}{c}
2 n \\
n
\end{array}\right]\right|_{q=r}=2 \ln q\left\{r+H_{(q)_{r}}^{(1)}-H_{(q)_{2 r}}^{(1)}\right\} \\
& \left.\frac{d^{2}}{d n^{2}} \ln \left[\begin{array}{c}
2 n \\
n
\end{array}\right]\right|_{q=r}=4(\ln q)^{2} \\
& \cdot\left[H_{(q)_{2 r}}^{(1)}-H_{(q)_{2 r}}^{(2)}+\frac{1}{2}\left\{H_{(q)_{r}}^{(2)}-H_{(q)_{r}}^{(1)}\right\}+\frac{1}{2} S(q, 2)\right] .
\end{aligned}
$$

By virtue of Bell polynomials we can derive further

$$
\begin{aligned}
& \frac{d^{2}}{d n^{2}}\left[\begin{array}{c}
2 n \\
n
\end{array}\right]_{q}=\left[\begin{array}{c}
2 n \\
n
\end{array}\right]_{q=r}\left[\left\{r+H_{(q)_{r}}^{(1)}-H_{(q)_{2 r}}^{(1)}\right\}^{2}\right. \\
& \left.\quad+\frac{1}{2} S(q, 2)+H_{(q)_{2 r}}^{(1)}-H_{(q)_{2 r}}^{(2)}+\frac{1}{2}\left(H_{(q)_{r}}^{(2)}-H_{(q)_{r}}^{(1)}\right)\right] .
\end{aligned}
$$

Finally plugging back the above identity in (110) we can obtain Theorem 19.

\section{Conclusion}

We would like to enrich the subject by doing further research in this field. In our upcoming paper, we will use the theorems of this paper to prove some series identities related to the Hurwitz Zeta function and also generalize a new theorem. Also we will derive some closed form identities related to double differentiation of Legendre Polynomials with respect to their order and we will derive some new generating function identities involving generalized harmonic numbers and Stirling polynomials.

By using the same techniques derived in this paper one can find various hypergeometric transformation formulas and their $q$-analog. We will consider a curious generalization expression such as

$$
\sum_{v=1}^{\infty} \frac{\left\{(q)_{v-1}\right\}^{m}}{\left\{(q)_{r+v}\right\}^{m}}\left(a q^{r}\right)^{v}
$$

in our upcoming paper.

\section{Competing Interests}

The author declares that there are no competing interests regarding the publication of this paper.

\section{Acknowledgments}

The author is grateful to Dr. Mourad Ismail for his careful comments and suggestions.

\section{References}

[1] E. W. Barnes, "The asymptotic expansion of integral functions defined by generalised hypergeometric series," Proceedings of the London Mathematical Society, vol. 2-5, no. 1, pp. 59-116, 1907.
[2] W. Maier and L. J. Slater, "L. J. Slater, Generalized Hypergeometric Functions. XIII +273 S. m. Fig. u. Tab. Cambridge 1966. University Press. Preis geb. 70 s. net," Journal of Applied Mathematics and Mechanics, vol. 46, no. 5, p. 332, 1966.

[3] R. L. Graham, D. E. Knuth, and O. Patashnik, Concrete Mathematics: A Foundation for Computer Science, Addison-Wesley, Boston, Mass, USA, 2nd edition, 1994.

[4] D. Connon, "Euler-hurwitz series and non-linear euler sums," https://arxiv.org/abs/0803.1304.

[5] S. M. Ripon, "Generalization of harmonic sums involving inverse binomial coefficients," Integral Transforms and Special Functions, vol. 25, no. 10, pp. 821-835, 2014.

[6] S. M. Ripon, "Generalization of a class of logarithmic integrals," Integral Transforms and Special Functions, vol. 26, no. 4, pp. 229245, 2015.

[7] W. Wang and C. Jia, "Harmonic number identities via the Newton-Andrews method," The Ramanujan Journal, vol. 35, no. 2, pp. 263-285, 2014.

[8] K. N. Boyadzhiev, "Harmonic number identities via euler's transform," Journal of Integer Sequences, vol. 12, no. 6, Article ID 09.6.1, 8 pages, 2009.

[9] H. Liu and W. Wang, "Harmonic number identities via hypergeometric series and Bell polynomials," Integral Transforms and Special Functions, vol. 23, no. 1, pp. 49-68, 2012.

[10] J. Wang and C. Wei, "Derivative operator and summation formulae involving generalized harmonic numbers," Journal of Mathematical Analysis and Applications, vol. 434, no. 1, Article ID 19793, pp. 315-341, 2016.

[11] P. Paule and C. Schneider, "Computer proofs of a new family of harmonic number identities," Advances in Applied Mathematics, vol. 31, no. 2, pp. 359-378, 2003.

[12] W. Chu and L. De Donno, "Hypergeometric series and harmonic number identities," Advances in Applied Mathematics, vol. 34, no. 1, pp. 123-137, 2005.

[13] H. Gould, Combinatorial Identities: A Standardized Set of Tables Listing 500 Binomial Coefficient Summations, West Virginia University, Morgantown, WVa, USA, 1972, https://books.google .com/books?id=idI-AAAAIAAJ.

[14] H. Exton, "A new two-term relation for the ${ }_{3} \mathrm{~F}_{2}$ hypergeometric function of unit argument," Journal of Computational and Applied Mathematics, vol. 106, no. 2, pp. 395-397, 1999.

[15] W. N. Bailey, "On the sum of a terminating ${ }_{2} \mathrm{~F}_{2}$," The Quarterly Journal of Mathematics, vol. 4, no. 1, pp. 237-240, 1953.

[16] A. K. Rathie, Y. S. Kim, and H. K. Song, "Another method for a new two-term relation for the hypergeometric function ${ }_{3} F_{2}$ due to Exton," Journal of Computational and Applied Mathematics, vol. 167, no. 2, pp. 485-487, 2004.

[17] P. J. Larcombe, E. J. Fennessey, and W. A. Koepf, "Integral proofs of two alternating sign binomial coefficient identities," Utilitas Mathematica, vol. 66, pp. 93-103, 2004.

[18] J. Choi and H. M. Srivastava, "A certain family of series associated with the zeta and related functions," Hiroshima Mathematical Journal, vol. 32, no. 3, pp. 417-429, 2002.

[19] D. Zeilberger, "Gauss's ${ }_{2} F_{1}(1)$ cannot be generalized to ${ }_{2} F_{1}(x)$," Journal of Computational and Applied Mathematics, vol. 39, no. 3, pp. 379-382, 1992.

[20] J. Wimp, “The computation of ${ }_{3} F_{2}(1)$," International Journal of Computer Mathematics, vol. 10, no. 1, pp. 55-62, 1981.

[21] J. Wimp, "Irreducible recurrences and representation theorems for ${ }_{3} F_{2}(1)$," Computers \& Mathematics with Applications, vol. 9, no. 5 , pp. $669-678,1983$. 
[22] A. Dil and V. Kurt, "Polynomials related to harmonic numbers and evaluation of harmonic number series II," Applicable Analysis and Discrete Mathematics, vol. 5, no. 2, pp. 212-229, 2011.

[23] L. J. Rogers, "Second memoir on the expansion of certain infinite products," Proceedings of the London Mathematical Society, vol. s1-25, no. 1, pp. 318-343, 1893.

[24] G. Andrews, R. Askey, and R. Roy, Special Functions, Encyclopedia of Mathematics and Its Applications, Cambridge University Press, 1999, https://books.google.com/books?id=qZWuYQbpkdMC.

[25] G. E. Andrews, "On the proofs of the Rogers-Ramanujan identities," in q-Series and Partitions, vol. 18 of Institute for Mathematics and Its Applications Volumes in Mathematics and Its Applications, pp. 1-14, Springer, New York, NY, USA, 1989. 


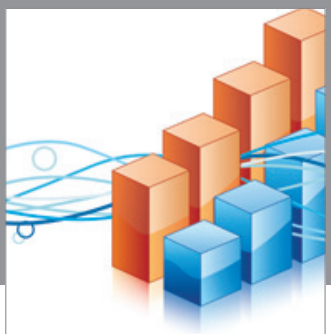

Advances in

Operations Research

vatem alat4

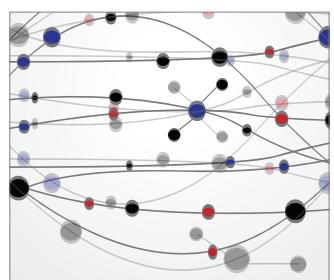

\section{The Scientific} World Journal
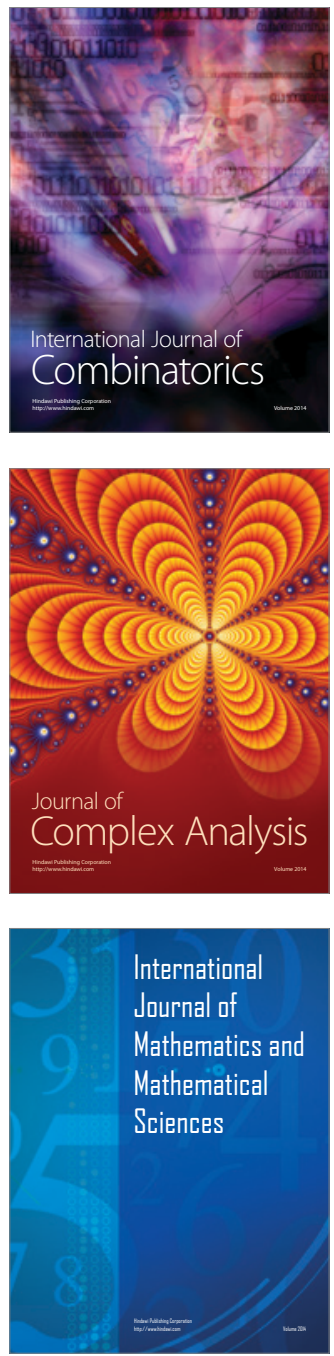
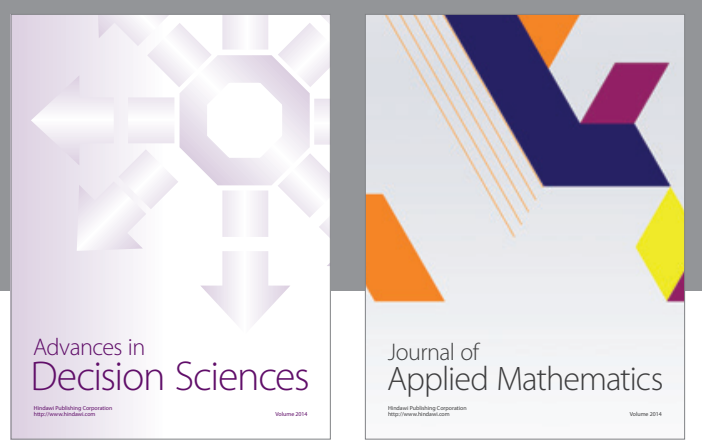

Algebra

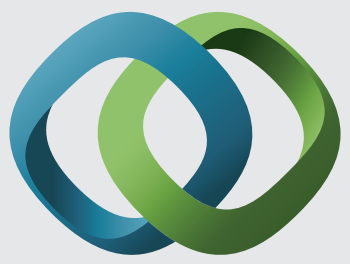

\section{Hindawi}

Submit your manuscripts at

http://www.hindawi.com
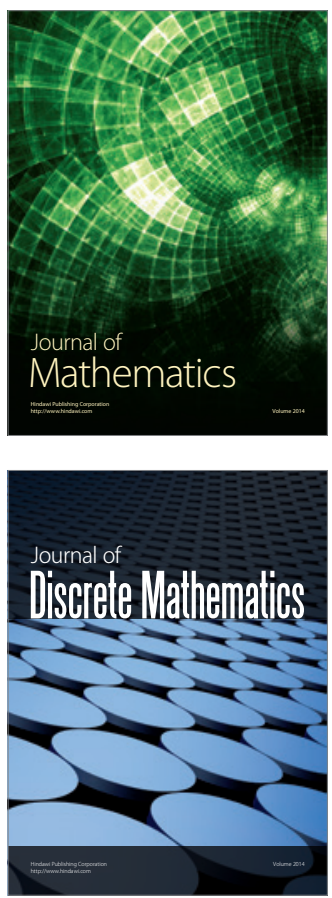

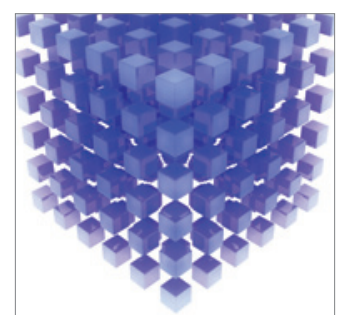

Mathematical Problems in Engineering
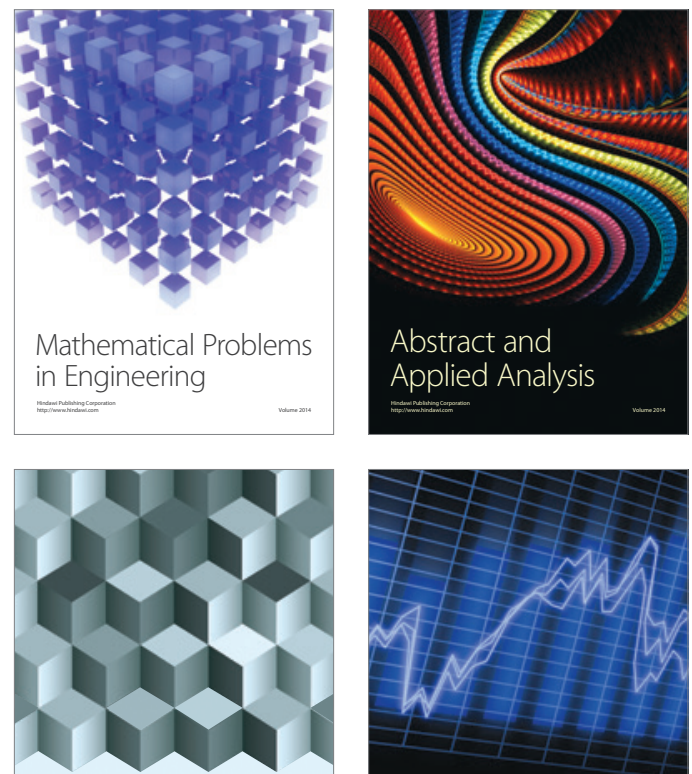

Journal of

Function Spaces

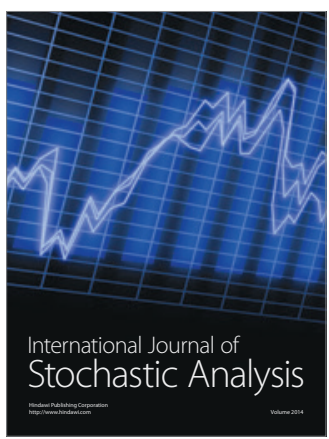

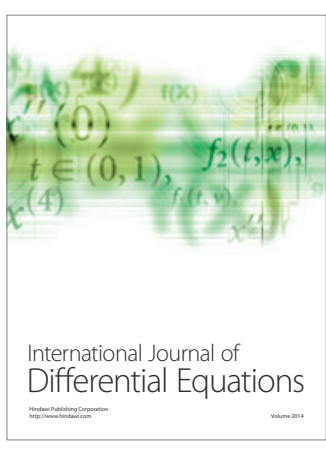
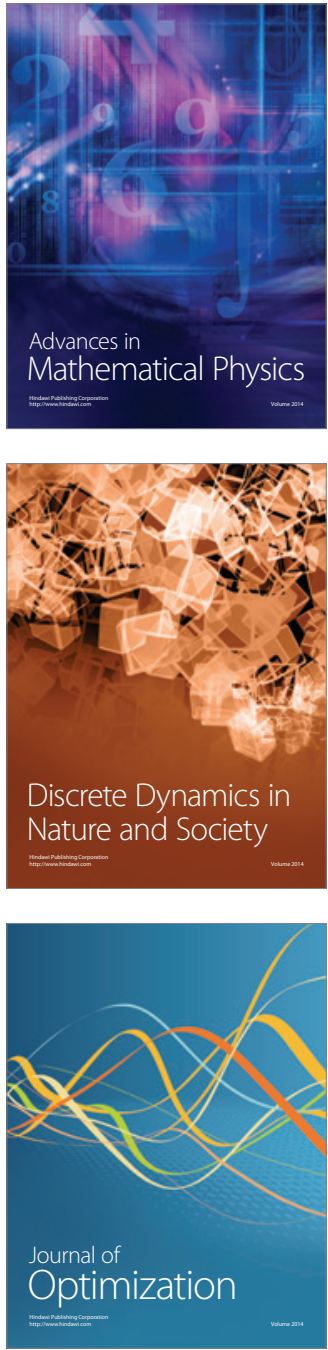